\title{
Update on Clinical Strategies in Hereditary Hemorrhagic Telangiectasia from an ENT Point of View
}

\author{
Kornelia E. C. Wirsching · Thomas S. Kühnel \\ Department of Otorhinolaryngology, University of Regensburg, Regensburg, Germany
}

Objectives. Hereditary hemorrhagic telangiectasia (HHT) is characterized by the presence of vascular malformations with an absence of capillaries between arteries and veins. One major manifestation site is the nasal mucous membrane where recurrent nosebleeds occur. Our clinical strategy to treat patients with HHT has the aim to reduce nasal bleeding long-term with minimal local and general side effects.

Methods. We describe staged diagnosis and therapy including individual medical treatments of 97 patients with HHT. The success of treatment is monitored with a systematic questionnaire.

Results. The neodymium-doped yttrium aluminium garnet (Nd:YAG) laser therapy remains standard treatment of choice with no major side effects despite the need for repeated treatment. In addition new treatment strategies like nasal occlusion, local drug therapy, and nasal septal splinting show initial success.

Conclusion. Improvement of the quality of life of HHT patients can be achieved by a multimodal concept. Several new treatment strategies like nasal septal splinting and nasal occlusion successfully expand the range of established methods. Further studies have to prove the safety and long-term effectiveness of the described individual medical treatments.

Keywords. Epistaxis; Hereditary Hemorrhagic Telangiectasia; Osler-Rendu-Weber Syndrome; Arteriovenous Malformations; Nd:YAG Laser; Bevacizumab

\section{INTRODUCTION}

Hereditary hemorrhagic telangiectasia (HHT; Rendu-Osler-Weber syndrome) is an autosomal dominant disorder causing vascular malformations in mucocutaneous tissues, visceral organs, and the central nervous system. The underlying pathology is a defect in the vascular wall. The development of these malformations starts with dilation of postcapillary venules and leads to expansion of the upstream vessels [1]. In the end arteriovenous

\footnotetext{
- Received February 19, 2016

Revised May 11, 2016

Accepted May 30, 2016

- Corresponding author: Kornelia E. C. Wirsching

Department of Otorhinolaryngology, University of Regensburg, Franz-Josef-

Strauss-Allee 11, Regensburg D-93053, Germany

Tel: +49-941-944-9410, Fax: +49-941-944-9405

E-mail: Kornelia.Wirsching@ukr.de
}

shunts develop. Diagnostic criteria, referred to as the Curaçao criteria, include epistaxis, telangiectases, visceral lesions, and affected first degree relatives. Epistaxis is a major manifestation of HHT affecting almost $100 \%$ of the patients by the age of 40 [2]. The intensity of this symptom varies from mild, scarce bleeding to life threatening epistaxis requiring nasal packing, hospitalization, and frequent blood transfusion limiting the patients quality of life. With an incidence of $1: 10,000$ HHT belongs to the so called orphan diseases being mainly treated in specialized centers [3-6]. The aim of the following study was to describe our staged diagnosis and individual treatment strategies as well as to demonstrate our results.

Copyright (C) 2017 by Korean Society of Otorhinolaryngology-Head and Neck Surgery.

This is an open-access article distributed under the terms of the Creative Commons Attribution Non-Commercial License (http://creativecommons.org/licenses/by-nc/4.0)

which permits unrestricted non-commercial use, distribution, and reproduction in any medium, provided the original work is properly cited. 
Table 1. Curaçao criteria

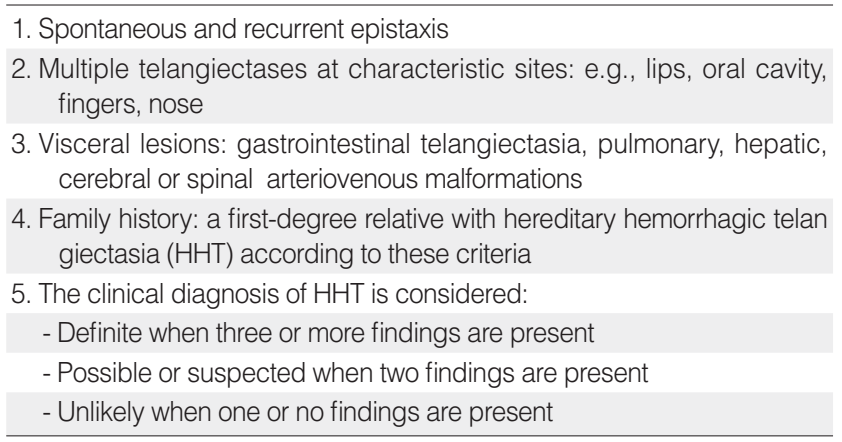

been treated in our ENT Clinic at the University of Regensburg. Ninety-seven patients are currently treated on a quarterly or at least annual basis. This article states our recent protocol of staged diagnosis and treatment of patients with HHT. The mean age of our patients is 58 years the ratio between male and female patients $47: 50$. The oldest patient is 88 years and the youngest patient 14 years. The average number of treatments was 16 , ranging from 1 to 67 .

At primary presentation we attempt to get a detailed picture of the patient. This includes past medical history as well as a detailed examination emphasizing on the possible manifestation sites of the disease. The diagnosis is based on clinical features referred to as Curaçao criteria (Table 1). The diagnosis is definite if 3 to 4 of the criteria match with a positive predictive value of $100 \%$ [7]. At the patients request molecular-genetical testing is available to contribute to establish the diagnosis. A genetic counselling session is legally required in advance. The second essential element of first consultation is providing comprehensive information on HHT. Following international recommendations a whole-body examination including the following diagnostic methods is suggested [2]: at the time of diagnosis blood tests for hemoglobin, ferritin and transaminases, contrast-enhanced echocardiogram and doppler ultrasound of the liver is recommended. Furthermore performing magnetic resonance imaging of the brain is offered. Patients over 35 years with suspected gastrointestinal (GI) bleeding are advised to have a GI endoscopic investigation. We repeat blood testing at least annually. Recommendation for follow-up examinations depend on the

\section{H I G H L I G G H T S}

- Neodymium-doped yttrium aluminium garnet (Nd:YAG) laser therapy remains the golden standard in treatment of epistaxis in hereditary hemorrhagic telangiectasia (HHT) patients.

- Nasal occlusion, local drug therapy, and nasal septal splinting are showing first positive effects on epistaxis in HHT patients.

- A multimodal and individual treatment concept is necessary to improve HHT patients quality of life.
Table 2. Hereditary hemorrhagic telangiectasia questionnaire

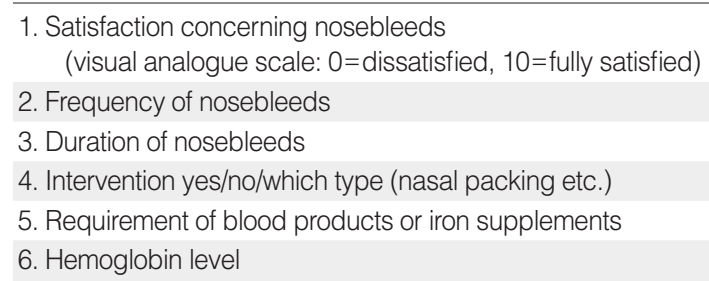

clinical findings.

The success of the patient-specific therapy is monitored with a systematic questionnaire in the beginning of each consultation. Adjustments of therapy depend on the answers and the patients individual wish for therapy (Table 2).

Instructions for regular nasal mucosal care include ointment and temporary closure of the nose. Nasal occlusion with adhesive, hypoallergenic tape (1 inch; 3M Micropore) for several hours per day achieves a moist nasal mucosa, promotes wound healing and possibly prevents the development of new Osler pits. A length of 2.4 inches is sufficient to close both nostrils. A case-control study on the use of hypoallergenic tape for nasal occlusion has been approved by the Ethics Committee of the University of Regensburg.

The core module of treatment is the neodymium-doped yttrium aluminium garnet (Nd:YAG) laser therapy under local anaesthesia at intervals ranging from 2 to 12 months. All of our 97 patients received this treatment. We use a pulsed Nd:YAG laser system manufactured by Fa DEKA-LMS Laser (El.En. Group, Calenzano, FL, Italy) into which a $200-\mu \mathrm{m}$ fiber is coupled. The treatment process has been described in detail in previously published documents [8]. Acute bleeding is managed with a radiofrequency generator (BM-780 II, Sutter Medical Group, Freiburg, Germany) with a nonstick forceps in bipolar coagulation mode at lowest possible energy setting. Usually $1.5 \mathrm{~W}$ give favourable results.

An important element of consultation is the instruction for self-care. This includes practicing the application of ointment, nasal tape or nasal packing. To prepare the patients for the event of acute epistaxis we provide a graduated scheme for self tamponade.

Patients who can not be treated sufficiently with Nd:YAG laser are offered local treatment with bevacizumab (Avastin, Roche) off-label as an individual treatment attempt. For this purpose $3.75 \mathrm{mg}$ bevacizumab diluted to $1 \mathrm{~mL}$ saline is injected submucosally in the anterior parts of the nose (cartilaginous septum/inferior turbinate, nasal floor, lateral nasal wall). Safety and effect of the local treatment with bevacizumab on the basis of an retrospective analysis of 11 patients with the approval of the local ethics committee has been published earlier [9].

If intensity of bleeding increases despite the management described above, endonasal treatment under general anaesthesia is considered. Compatibility tested blood reserves are prepared for 
the patients before the operation and stored in our blood depot. In the first step of the operation neuro-cotton wool soaked in epinephrine 1:1,000 is inserted in the nose for 10 minutes, provided that cardiovascular disease has been excluded. Thereafter endonasal coagulation of the sphenopalatine artery is performed endoscopically by radiofrequency generator on either side of the nose. Despite the knowledge of the benefits of that procedure being short-lived it serves the purpose to control intraoperative bleeding $[10,11]$. Subsequently all telangiectatic foci are treated endoscopically with Nd:YAG laser. In case of acute bleeding or vessels to large to respond adequately to laser treatment we use radiofrequency coagulation. Large arteriovenous shunts located in lateral nasal wall are covered with a ready-to-use haemostatic, collagen patch (TachoSil, Baxter Healthcare Co., Westlake Village, CA, USA). At the end of the procedure silicone splints with maximum surface coverage of the nasal septum are inserted and fixed with trans-septum suturing. The septal splints remain intranasally as long as they are comfortable for the patient. Nasal irrigation is recommended to keep the splints clear from bacteria and fungus growth.

\section{RESULTS}

To prove the effectiveness and success of our treatment strategy we designed a systematic questionnaire (Table 2, Fig. 1). In the majority of cases a decrease of epistaxis is seen after one or two laser sessions. Taking into account that the arteriovenous (AV) malformations are continuously re-emerging, treatment intervals of 3 to 6 months are recommended. Fig. 1 displays the development of bleeding and subjective satisfaction of our latest 10 patients being treated 2-4 times with the Nd:YAG laser. Especially patients with mild to moderate disease benefit from regular laser irradiation. The frequency of nosebleeds decreased from 7 times per week to 2 times per week. The duration of nosebleeds was stated 9 minutes initially and 4 minutes after 2-4 times of

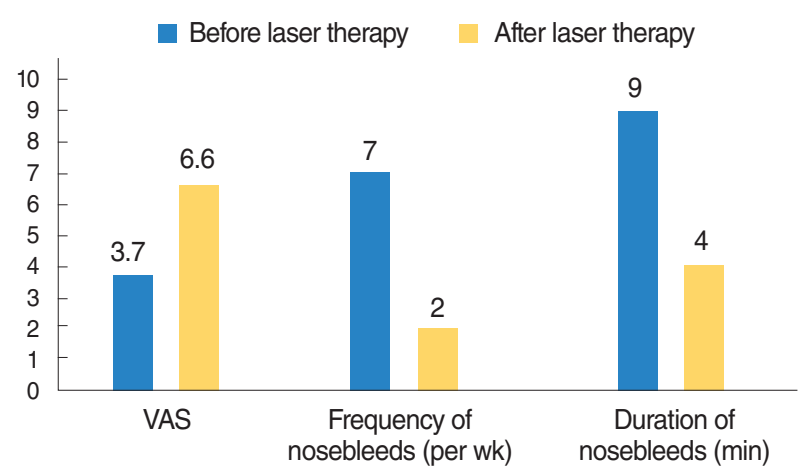

Fig. 1. Comparison of answers to the hereditary hemorrhagic telangiectasia questionnaire of the latest 10 patients being treated 2-4 times with the neodymium-doped yttrium aluminium garnet (Nd:YAG) laser. VAS, visual analogue scale. laser treatment. The Nd:YAG laser coagulates the telangiectatic foci submucosally without causing permanent damage of the overlying mucosal layer. To date no relevant scarring or septal defect has occured in our patient population after regular therapy. Occlusion of the nostrils by tape for several hours a day seems to further help to prevent the recurrence of mucosal AV shunts. Initial observations show that after 3 months of regular nasal occlusion nasal crusting and the intensity and frequency of nasal bleeding decrease.

As published earlier, additional application of $3.75 \mathrm{mg}$ bevacizumab submucosally has shown a positive effect on frequency and severity of epistaxis [9]. In context of the retrospective analysis quoted above we have not seen complications like atrophy of the mucosa or septal perforation.

A proportion of our patients suffer from severe recurrent epistaxis requiring blood transfusions. In these cases treatment under general anaesthesia is beneficial. Large vascular protuberances can not be irradiated by Nd:YAG laser because of high blood flow. These sites are coagulated with nonstick radiofrequency forceps (1.5-3 W). In order to minimize intraoperative bleeding both sphenopalatine arteries are obliterated at the beginning of surgery. AV shunts located in the lateral nasal wall can sometimes be very large with the apparent spot just being the tip of the iceberg. Covering those AV malformations with a haemostatic collagen patch (TachoSil) after coagulation has helped to improve the final result of surgery in severe cases. By now 16 patients have received silicone nasal septal splints offering maximum possible septum coverage at the end of the operation. The majority of patients report a strong decline of epistaxis postoperatively. Fig. 2 shows the duration and frequency of nosebleeds of our latest 6 patients before and 2 to 3 months after receiving this treatment. The frequency of nosebleeds decreased from 4 times per week to 2 times per week. The duration of nosebleeds decreased from 22 minutes to 3 minutes. The maximum duration of application by now is 24 months. In 4 patients the septal splints had to be removed because of once dis-

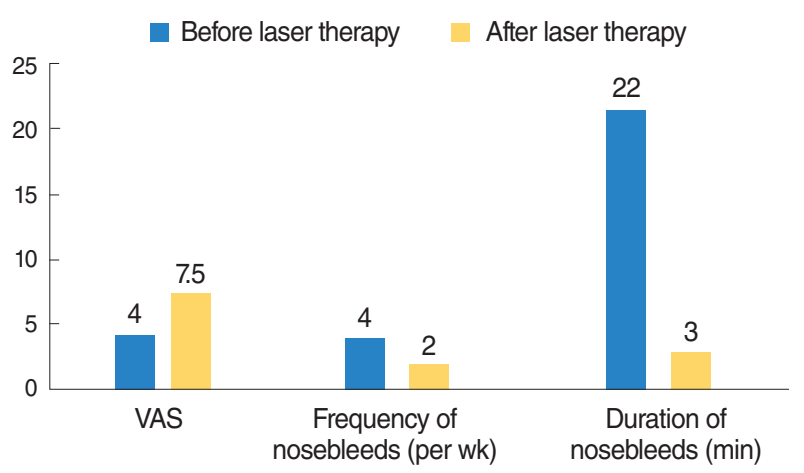

Fig. 2. Comparison of answers to the hereditary hemorrhagic telangiectasia questionnaire of the latest 6 patients before and 2 to 3 months after receiving treatment after general anaesthesia including septal splints. VAS, visual analogue scale. 
comfort, twice fungal growth on the splints. In one patient there was an increase of telangiectases on the edge of one splint leading to recurrent epistaxis.

\section{DISCUSSION}

Patients with HHT have been treated at the ENT University Clinic of Regensburg for over 20 years. Our treatment strategy is based upon extensive patient information, training courses for self care, prevention, treatment of epistaxis and comprehensive support for all disease specific problems. Besides established approaches the following discussion highlights new developments in italics.

The central element of the treatment is the Nd:YAG laser therapy which has proved to be an effective method to treat mucosal telangiectases since 34 years [11-13]. Over time the laser systems have improved in terms of precision thus reducing side effects. Effective coagulation of nasal vascular malformations under microscopic control at an internal power of 2-4 W minimally harm the surrounding mucosa a septal cartilage. In contrast to other medical centres we perform laser therapy in an outpatient setting under local anaesthesia without intravenous sedation [14-16]. This strategy saves the risk of recurrent general anaesthesia and is time effective for the patient. The local pain level is described as insignificant by the vast majority of patients. After up to 67 single laser procedures in a collective of 97 patients that are currently treated by Nd:YAG laser we have not seen severe scarring or septal perforation. The assessment of the visual analogue scale (VAS) on the satisfaction concerning the patients nosebleeds shows an improvement after 2 to 4 outpatient treatments (Fig. 1). Yet one has to consider that there is a lack of controlled studies on this topic. Therefore our subjective experience is a major component developing our actual treatment strategy. A statement why we selected the Nd:YAG laser out of a number of local treatment strategies has been discussed earlier [8].

The application of soft ointment for lubrication of the nasal mucosa is an established part of basic nasal care having a preventive effect on nasal bleeding [15]. Within the last years focus was set on further options to avoid of the development of nasal telangiectasia. The Young`s procedure (permanent surgical closure of the nostrils) prevents the so called "second hit" for the development of AV malformation by stopping the air flow, leading to a complete cessation of epistaxis [17]. Taking a loss of severe side effects like permanent blocked nose, xerostomia, anosmia or nasal voice this treatment remains a stopgap for patients with very serious epistaxis. Intermittent nasal occlusion by tape produces a moist chamber and prevents nasal airflow. The daily length of treatment is determined by the patient. The hypoallergenic tape is inexpensive, individually adaptable, simple to apply and remove. We consider this an effective method for nasal mu- cosal care without the considerable disadvantages of permanent nasal occlusion. Quantification of the effect on the frequency and intensity of nasal bleeding shall be displayed in future. Blocking the nose with cotton wool soaked with ointment is a popular technique of Morbus Osler patients to avoid or treat nasal bleeding. Compared to nasal occlusion by tape there is a risk of rupurting AV shunts in the nasal vestibulum during application. Sometimes the cotton wool cannot be removed completely. Remnants can lead to sneezing, crusting or mucosal infections. Another interesting approach of nasal occlusion are custom-made silicone olives for each nostril. Yet they require cost and time-intensive individual adjustment and repeated manipulation in the nasal vestibulum which is unfavorable in Morbus Osler patients in our personal experience [18].

A further option of therapy that has become increasingly important is the local and systemic drug administration. The level of vascular endothelial growth factor (VEGF) is elevated in patients with HHT [19]. The injection of the VEGF-antibody bevacizumab submucosally in addition to the Nd:YAG laser therapy leads to a better subjective outcome in first retrospective studies [9]. We offer this treatment as an off-label use to patients who do not respond positively to the laser therapy. Future studies will reveal the role of local and systemic drug treatment for HHT patients.

There are also HHT patients who do not sufficiently benefit from Nd:YAG laser therapy under local anaesthesia. This applies to patients with large vascular protuberances especially in the posterior portion of the nose, leading to severe epistaxis and requiring blood transfusions. In an operation under general anesthesia the affected Osler sites are treated with Nd:YAG laser or radiofrequency coagulation. To reduce the risk of intraoperative bleeding both sphenopalatine arteries are coagulated. Contrary to the prevalent method of vessel clipping for posterior epistaxis we consciously choose the method of coagulation of both sphenopalatine arteries in patients with HHT. Coagulated vessels recanalize over time. The main feeder of the nasal mucosal blood supply therefore remains in the area around the ethmoidal crest and can be found and obliterated again in case of further operations. In contrast, vessel clips ligate the sphenopalatine artery permanently and circulatory bypasses develop. According to our experience collateral vessels are not as well anatomically defined as is the sphenopalatine artery. Thus seeking and obliteration of these vessels is challenging.

As described above, telangiectatic foci in the lateral wall of the nose can develop to extensive AV shunts. Endonasal treatment is limited to the surface of the nasal mucosa. Therefore we cover the area of large AV shunts in the lateral wall of the nose with haemostatic, collagen fleece (TachoSil) after coagulation in order to minimize mucosal lesions and acute bleeding of this vulnerable area. Yet, this technique is the result of our clinical experience and has not been described or analyzed in literature. An established technique to treat large AV shunts being fed by 
maxillary or facial artery is transcatheter embolization [20,21]. Due to development of collateral vessels cessation of bleeding is only temporary. Complications of this radiological intervention include skin necrosis, ipsilateral blindness and stroke [22]. Therefore we consider transcatheter embolization only in case of life threatening epistaxis in HHT patients.

At the end of the procedure nasal septal splints, covering the major part of the septum are sewed in. Again this prevents nasal air flow being the "second hit" for the development of new telangiectasia. Compared to permanent nasal occlusion nasal airflow is partly preserved. Moreover the septal splints can be removed easily on request of the patient. Frequency and intensity of epistaxis decreased in 14/16 patients after the operation with a maximum follow-up of 24 months. To the best of our knowledge optimised design splints should have the following characteristics: extensive coverage of the nasal septum, atraumatic design with no contact with the roof, floor, lateral parts of the nose and base of septum and a special surface coverage to prevent germinal growths (i.e., silver ions).

Being part of the center for orphan diseases (ZSER, University Clinic of Regensburg, Regensburg; zser@ukr.de, http://www. zser.de) the University Clinic of Regensburg is able to supply relevant expertise to manage all aspects of Morbus Osler. Furthermore interested patients are introduced to the "Morbus Osler support group" (Morbus Osler Selbsthilfe e.V., Niederkruechten, Germany; http://www.morbus-osler.de). This central platform offers an exchange of knowledge between patients and experts and psychological support.

There has been a great demand for optimizing and bundling the treatment of patients with hereditary hemorrhagic telangiectasia. Our approach to treat the local manifestations of HHT with Nd:YAG laser and offer a multimodal medical support for this group of patients performs well in terms of reduction of nosebleeds and improvement of their quality of life.

\section{CONFLICT OF INTEREST}

No potential conflict of interest relevant to this article was reported.

\section{REFERENCES}

1. Braverman IM, Keh A, Jacobson BS. Ultrastructure and three-dimensional organization of the telangiectases of hereditary hemorrhagic telangiectasia. J Invest Dermatol. 1990 Oct;95(4):422-7.

2. Faughnan ME, Palda VA, Garcia-Tsao G, Geisthoff UW, McDonald J, Proctor DD, et al. International guidelines for the diagnosis and management of hereditary haemorrhagic telangiectasia. J Med Genet. 2011 Feb;48(2):73-87.

3. Byahatti SV, Rebeiz EE, Shapshay SM. Hereditary hemorrhagic tel- angiectasia: what the otolaryngologist should know. Am J Rhinol. 1997 Jan-Feb;11(1):55-62.

4. Geisthoff UW, Schneider G, Fischinger J, Plinkert PK. Hereditary hemorrhagic telangiectasia (Osler's disease): an interdisciplinary challenge. HNO. 2002 Feb;50(2):114-28.

5. Guttmacher AE, Marchuk DA, White RI Jr. Hereditary hemorrhagic telangiectasia. N Engl J Med. 1995 Oct;333(14):918-24.

6. Porteous ME, Burn J, Proctor SJ. Hereditary haemorrhagic telangiectasia: a clinical analysis. J Med Genet. 1992 Aug;29(8):527-30.

7. van Gent MW, Velthuis S, Post MC, Snijder RJ, Westermann CJ, Letteboer TG, et al. Hereditary hemorrhagic telangiectasia: how accurate are the clinical criteria? Am J Med Genet A. 2013 Mar;161 (3):461-6.

8. Kuhnel TS, Wagner BH, Schurr CP, Strutz J. Clinical strategy in hereditary hemorrhagic telangiectasia. Am J Rhinol. 2005 Sep-Oct;19 (5):508-13.

9. Rohrmeier C, Sachs HG, KuehnelTS. A retrospective analysis of low dose, intranasal injected bevacizumab (Avastin) in hereditary haemorrhagic telangiectasia. Eur Arch Otorhinolaryngol. 2012 Feb;269 (2):531-6.

10. Golding-Wood PH. The role of arterial ligation in intractable epistaxis. J Laryngol Otol Suppl. 1983;8:120-2.

11. Werner JA, Geisthoff UW, Lippert BM, Rudert H. Treatment of recurrent epistaxis in Rendu-Osler-Weber disease. HNO. 1997 Sep;45 (9):673-81.

12. Parkin JL, Dixon JA. Laser photocoagulation in hereditary hemorrhagic telangiectasia. Otolaryngol Head Neck Surg. 1981 MarApr;89(2):204-8.

13. Shapshay SM, Oliver P. Treatment of hereditary hemorrhagic telangiectasia by Nd-YAG laser photocoagulation. Laryngoscope. 1984 Dec;94(12 Pt 1):1554-6.

14. Kluger PB, Shapshay SM, Hybels RL, Bohigian RK. NeodymiumYAG laser intranasal photocoagulation in hereditary hemorrhagic telangiectasia: an update report. Laryngoscope. 1987 Dec;97(12): 1397-401.

15. Folz BJ, Tennie J, Lippert BM, Werner JA. Natural history and control of epistaxis in a group of German patients with Rendu-OslerWeber disease. Rhinology. 2005 Mar;43(1):40-6.

16. Mahoney EJ, Shapshay SM. New classification of nasal vasculature patterns in hereditary hemorrhagic telangiectasia. Am J Rhinol. 2006 Jan-Feb;20(1):87-90.

17. Gluckman JL, Portugal LG. Modified Young's procedure for refractory epistaxis due to hereditary hemorrhagic telangiectasia. Laryngoscope. 1994 Sep;104(9):1174-7.

18. Al Kadah B, Papaspyrou G, Schneider M, Schick B. First experiences with an individual nasal olive in patients with hereditary haemorrhagic telangiectasia (HHT). Eur Arch Otorhinolaryngol. 2015 Jan; 272(1):117-22.

19. Sadick H, Riedel F, Naim R, Goessler U, Hormann K, Hafner M, et al. Patients with hereditary hemorrhagic telangiectasia have increased plasma levels of vascular endothelial growth factor and transforming growth factor-beta1 as well as high ALK1 tissue expression. Haematologica. 2005 Jun;90(6):818-28.

20. Fischer M, Dietrich U, Labisch C, Zanella FE, Jahnke K. Critical evaluation of vascular embolization in patients with Rendu-Osler disease. Laryngorhinootologie. 1997 Aug;76(8):490-4.

21. Elden L, Montanera W, Terbrugge K, Willinsky R, Lasjaunias P, Charles D. Angiographic embolization for the treatment of epistaxis: a review of 108 cases. Otolaryngol Head Neck Surg. 1994 Jul;111 (1):44-50.

22. Dubel GJ, Ahn SH, Soares GM. Transcatheter embolization in the management of epistaxis. Semin Intervent Radiol. 2013 Sep;30(3): 249-62. 